Brit. Heart f., 1969, 31, 400.

\title{
Coronary Arteriovenous Fistula with Aneurysm of Coronary Sinus
}

\author{
ALAN HARRIS, KEITH JEFFERSON, AND KANU CHATTERJEE \\ From Cardiac Department, St. George's Hospital, London S.W.I
}

\begin{abstract}
Abnormal communications of the coronary vascular system are rare, the majority of instances having been described in children or young adults (Gasul et al., 1960; Papaioannou et al., 1962). The commonest vessel to be involved is the right coronary artery, and the communication is usually with the right heart chambers or the pulmonary artery.

A patient who was asymptomatic for 64 years, with a coronary artery fistula involving the left coronary artery and coronary sinus, is described.
\end{abstract}

\section{Case Report}

A 64-year-old housewife was admitted to hospital in February 1967, with a 2-week history of palpitation and orthopnoea. There was no previous history of any cardiac disease. On admission she had signs of left ventricular failure confirmed by the presence of pulmonary oedema on the $x$-ray film. Heart rate was 130 a minute, with multiple ventricular ectopic beats, and the left ventricle was hypertrophied. Blood pressure was $185 / 80 \mathrm{~mm} . \mathrm{Hg}$, jugular venous pressure normal, carotid pulse sharp, and there was a grade $3 / 6$ ejection systolic murmur maximal in the tricuspid area. No obvious cause was found for the left ventricular failure which rapidly improved with digitalis and diuretics. She was followed as an out-patient where she was seen to be free from heart failure with a normal exercise tolerance, but she continued to have episodes of palpitation lasting up to 30 minutes. During a routine out-patient attendance in April 1968, a continuous murmur was heard for the first time in the left axilla (Fig. 1). The carotid pulses were sharp, the left ventricle hypertrophied, and the blood pressure was $150 / 40 \mathrm{~mm}$. Hg. As signs suggested an arteriovenous shunt, she was admitted for cardiac catheterization and angiography to determine the site of the communication. At the time of investigation she weighed $74.8 \mathrm{~kg}$., height $173 \mathrm{~cm}$., and she was free from heart failure.

The chest $x$-ray film (Fig. 2) showed cardiac enlargement, cardiothoracic ratio $15 \cdot 8: 29 \cdot 3$. There was an angular projection at the junction of the middle and lower thirds of the left cardiac border. On the $x$-ray screening the small projection was best seen in the right anterior oblique position; this moved down and out with left ventricular systole, and was not typical of paradoxical pulsation seen in ventricular aneurysm. The electrocardiogram showed sinus rhythm, mean frontal axis $0^{\circ}$, left atrial hypertrophy, q lead I, aVL, V4-V7. Other routine investigations were normal.

Cardiac catheterization was carried out via a right antecubital fossa vein and right brachial arteriotomy. The patient was resting at the time of study and was not sedated. The resting pressure and cardiac output were normal (see Table), but during exercise there was a significant rise in the mean pulmonary capillary wedge and right ventricular pressures, suggesting impairment of left ventricular function. There was no evidence of a left-to-right intracardiac shunt on serial samples, but a blood sample was not obtained from the coronary sinus.

During left heart catheterization the catheter readily entered an enormously dilated left coronary artery.

Angiography. A No. 8 Mason Sones catheter was passed into the left coronary artery, and $25 \mathrm{ml}$. Urografin 76 was injected at a pressure of $30 \mathrm{lb}$./sq. in. Biplane Elema roll films were exposed at 6 a second.

The circumflex branch of the left coronary artery was very wide, measuring $1.5 \mathrm{~cm}$. in diameter. A large oval aneurysm $(8 \mathrm{~cm} . \times 7.5 \mathrm{~cm} . \times 6 \mathrm{~cm}$.) filled from the distal part of the circumflex artery (Fig. 3, 4, and 5). A filling defect within the aneurysm suggested the presence of a thrombus. The aneurysm corresponded in position to the proximal part of the coronary sinus. Later the distal part of the coronary sinus was opacified. It was widened but not aneurysmal. Finally the right atrium was outlined with contrast medium. No filling of the anterior descending branch of the left coronary artery occurred. This was probably due to a "steal" phenomenon as a result of high flow through the circumflex artery. A branch of the circumflex artery was seen to be dilated and tortuous and undoubtedly emptied into the aneurysm.

The grossly dilated proximal part of the circumflex branch corresponded to the angular projection on the left cardiac border on the plain chest $x$-ray (Fig. 2). The posterior bulge of the coronary sinus aneurysm could be identified on the lateral view of the plain chest $x$-ray. 


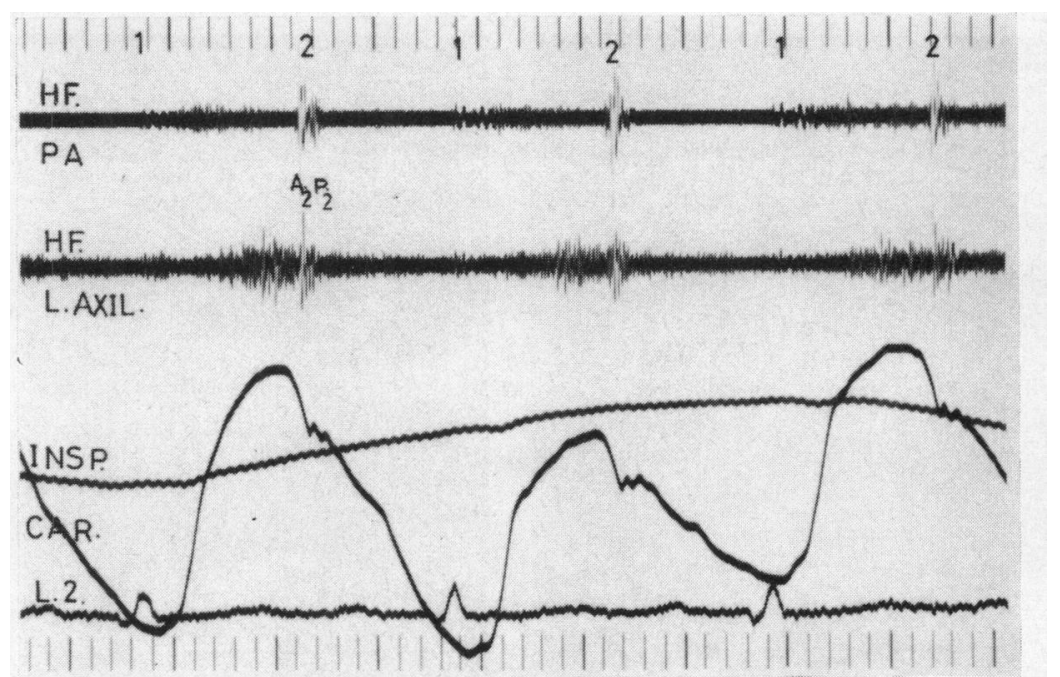

FIG. 1.-External phonocardiogram showing the continuous murmur which was localized to the left axilla. PA, pulmonary area; L. axil., left axilla; 1, first heart sound; 2, second heart sound; Insp., inspiration; L.2., lead II of electrocardiogram; HF, high frequency; CAR., external carotid pulse.

Intracardiac Phonocardiography. The continuous murmur was localized to the left coronary artery and was maximal well down the artery at the level of the large aneurysm. An ejection systolic murmur was recorded in the aortic root and left ventricular outflow tract.

Progress. Despite the tempting possibilities of a surgical cure, conservative management was recommended in the first instance in view of the patient's age, moderate obesity, and good symptomatic response to medical treatment.

\section{Discussion}

The radiological appearances in this patient correspond to the post-mortem findings in a patient reported by Davison, McCracken, and McIlveen (1955), where the circumflex branch of the left coronary artery was grossly dilated and communicated with the coronary sinus which was aneurysmal and formed a thin-walled bag $10 \mathrm{~cm}$. in diameter.

Coronary artery fistulae can usually be demonstrated by aortography (Newcombe, Whitaker, and

TABLE

CARDIAC CATHETERIZATION DATA

\begin{tabular}{|c|c|c|c|c|c|}
\hline \multirow{2}{*}{ Site } & \multicolumn{3}{|c|}{$\begin{array}{c}\text { Pressure (mm. Hg) measured from } \\
\text { sternal angle }\end{array}$} & \multicolumn{2}{|c|}{ Blood samples } \\
\hline & Systolic & Diastolic & Mean & $\begin{array}{c}\mathrm{O}_{2} \text { content } \\
\text { vol. per cent }\end{array}$ & $\mathrm{O}_{2}$ per cent \\
\hline $\begin{array}{l}\text { Pulmonary capillary wedge } \\
\text { Right pulmonary artery } \\
\text { Left pulmonary artery } \\
\text { Main pulmonary artery } \\
\text { High right ventricle } \\
\text { Low right atrium } \\
\text { Mid right atrium } \\
\text { High right atrium } \\
\text { Superior vena cava } \\
\text { Inferior vena cava }\end{array}$ & $\begin{array}{l}\overline{34} \\
34 \\
34 \\
31 \\
= \\
= \\
=\end{array}$ & $\begin{array}{l}\overline{14} \\
14 \\
14 \\
3 \\
= \\
= \\
=\end{array}$ & $\begin{array}{l}6 \\
24 \\
24 \\
24 \\
\overline{-} \\
3 \bar{a}=4 \\
=\end{array}$ & $\begin{array}{l}\overline{12 \cdot 6} \\
12 \cdot 3 \\
12 \cdot 3 \\
12 \cdot 5 \\
12 \cdot 0 \\
11 \cdot 6 \\
11 \cdot 1 \\
11 \cdot 3 \\
11 \cdot 3\end{array}$ & $\begin{array}{l}76 \\
74 \\
74 \\
75 \\
72 \\
70 \\
61 \\
68 \\
68\end{array}$ \\
\hline $\begin{array}{l}\text { Central aorta } \\
\text { Left ventricular cavity }\end{array}$ & $\begin{array}{l}150 \\
150\end{array}$ & $\begin{array}{r}70 \\
3\end{array}$ & - & $\begin{array}{l}16 \cdot 6 \\
16 \cdot 6\end{array}$ & $\begin{array}{l}98 \\
98\end{array}$ \\
\hline $\begin{array}{l}\text { Resting cardiac output } \\
\text { Oxygen uptake }\end{array}$ & \multicolumn{2}{|c|}{$\begin{array}{l}5.71 . / \mathrm{min} . \text { (Fick) } \\
246 \mathrm{ml} . / \mathrm{min} .\end{array}$} & 二 & & \\
\hline $\begin{array}{l}\text { Exercise-straight leg raising } \\
\text { Pulmonary capillary wedge } \\
\text { Right ventricle } \\
\text { Right atrium }\end{array}$ & $\underline{60}$ & $\overline{4}$ & $\frac{32}{7 a}=10$ & & \\
\hline
\end{tabular}




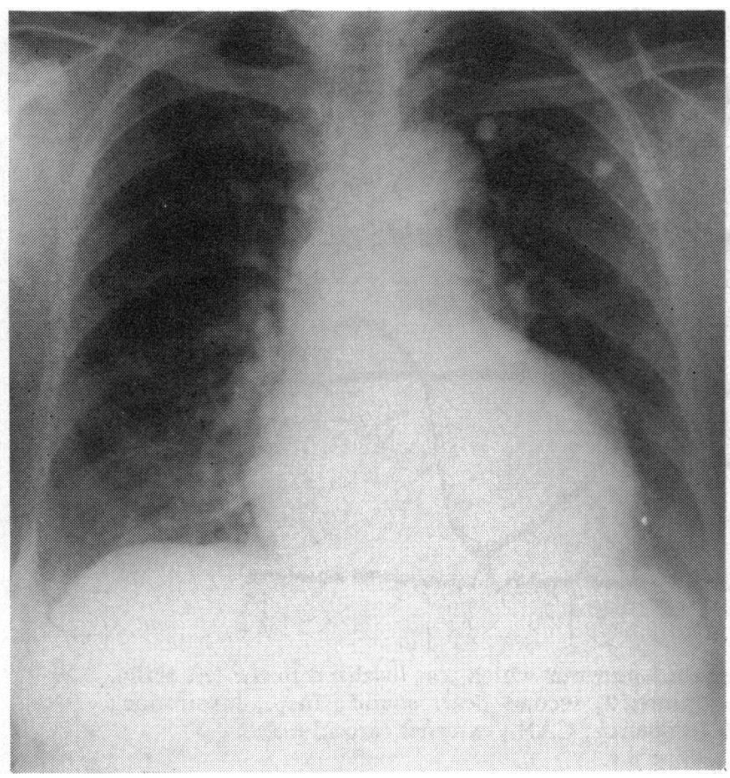

Fig. 2.-Postero-anterior chest film. Cardiac enlargement. Localized bulge on left cardiac border, subsequently shown to be due to a portion of the dilated circumflex branch of the left coronary artery.

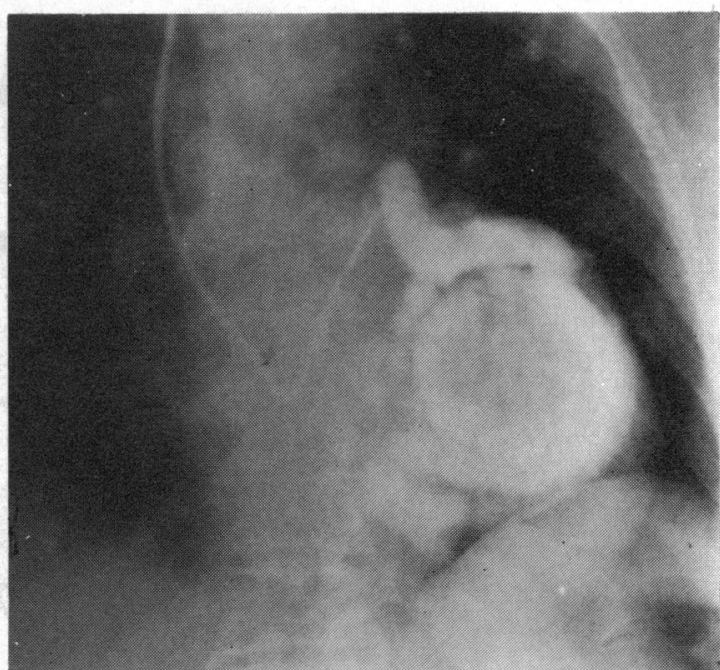

FIG. 3.-Selective injection into left coronary artery. The circumflex branch is grossly dilated. The aneurysm of the coronary sinus is beginning to fill.

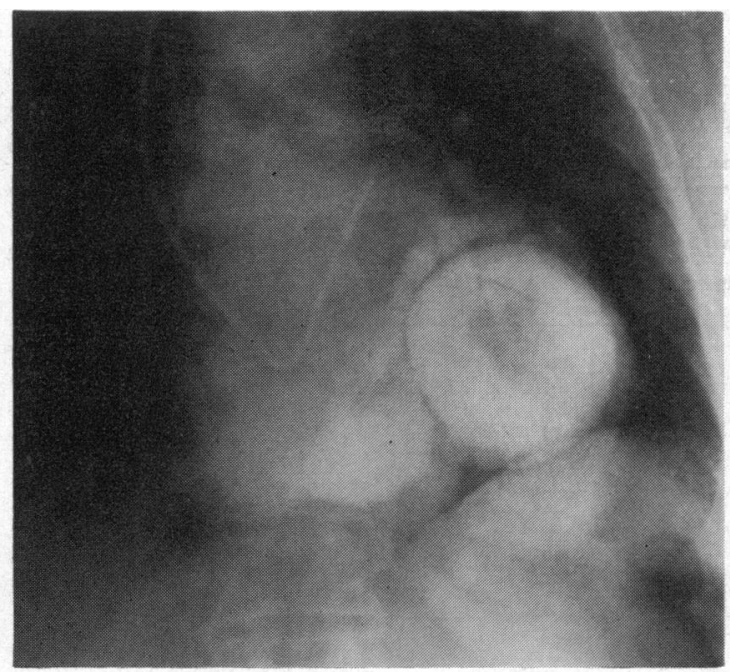

Fig. 4.- Half second later than Fig. 3. The coronary sinus aneurysm is densely opacified. A central filling defect suggests thrombus. The distal part of the coronary sinus is widened but not aneurysmal.

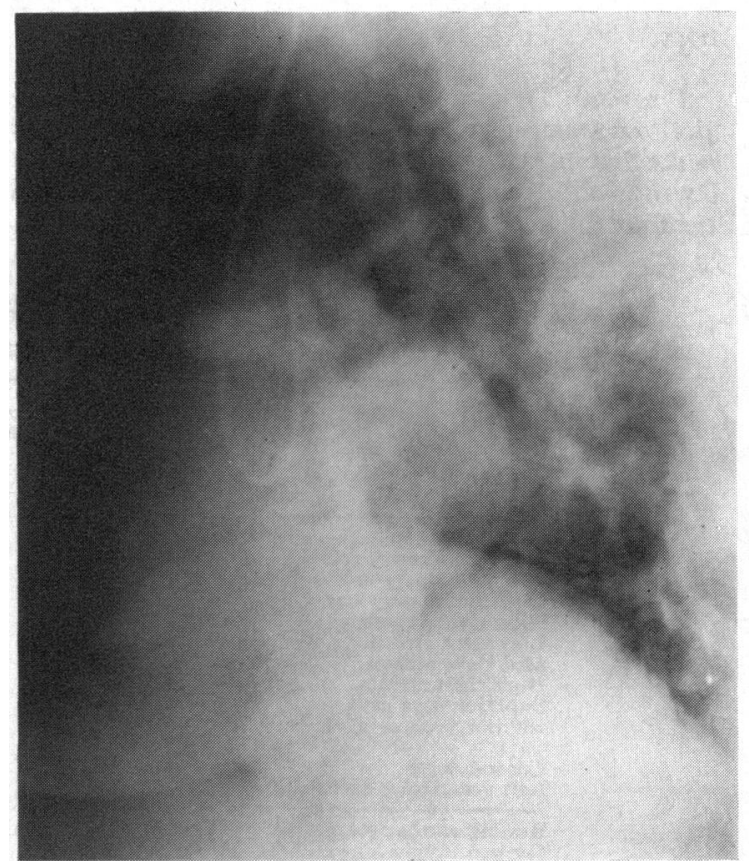

Fig. 5.-Lateral view is synchronous with Fig. 3. The aneurysm corresponds in position to the proximal part of the coronary sinus. The circumflex artery appears to be displaced somewhat forward by the aneurysm. 
Keates, 1964), but undoubtedly a better demonstration of the abnormal anatomy can safely be obtained with selective coronary angiography, by the introduction of a large volume of contrast medium injected under pressure.

The absence of a continuous murmur in our patient during her first admission to hospital was thought to be due to left ventricular failure. After successful treatment, a continuous murmur was detected in the left axilla, and it was of interest that the intracardiac phonocardiogram located the murmur within the left coronary artery at the level of the aneurysmal dilatation of the coronary sinus. In a third of patients with coronary arteriovenous fistula, the site of the continuous murmur is indistinguishable from that of a patent ductus (Gasul et al., 1960). However, in the remainder, the continuous murmur has an unusual location, as in this patient.

The absence of cardiac symptoms for 64 years in this patient indicates the benign course that coronary arteriovenous fistulae may run. Since surgical closure of the fistula may be difficult, we felt that we were not justified at present in recommending surgery, particularly with the marked symptomatic improvement obtained with digitalis and diuretic therapy.

\section{Summary}

A 64-year-old woman presented with clinical and radiological signs of left ventricular failure. After medical treatment a continuous murmur was heard in an unusual site (left axilla) and raised the possibility of a coronary arteriovenous fistula. Furthermore, the chest $x$-ray showed an abnormal bulge which corresponded well to the position of the murmur.

The fistula was demonstrated radiologically by selective coronary arteriography, with a pressure injection of a large volume of contrast into the left coronary artery, its circumflex branch communicating with an aneurysmal coronary sinus.

Intracardiac phonocardiography localized the continuous murmur to within the left coronary artery at the level of the aneurysmal coronary sinus.

\section{References}

Davison, P. H., McCracken, B. H., and McIlveen, D. J. S. (1955). Congenital coronary arterio-venous aneurysm. Brit. Heart f., 17, 569.

Gasul, B. M., Arcilla, R. A., Fell, E. H., Lynfield, J., Bicoff, J. P., and Luan, L. L. (1960). Congenital coronary arteriovenous fistula. Clinical, phonocardiographic, angiocardiographic and hemodynamic studies in five patients. Pediatrics, 25, 531.

Newcombe, C. P., Whitaker, W., and Keates, P. G. (1964). Coronary arterio-venous fistulae. Thorax, 19, 16.

Papaioannou, A., Agorogiannis, S., Nihoyanopoulos, J., and Lazzaridis, D. (1962). Congenital coronary artery fistula. Successful preoperative diagnosis and surgical correction. Amer. F. Cardiol., 10, 588. 